\title{
Determination of Compounds in Eucommia ulmoides Oliv. Bark and Its Fermentation Products via Headspace Gas Chromatography-ion Mobility Spectrometry
}

\begin{abstract}
Zhihong Wang, a,b Mijun Peng, a,* Zhigang She, ${ }^{\mathrm{b}, *}$ Yu Qiu, ${ }^{\mathrm{a}}$ Qiuling Yang, ${ }^{\mathrm{a}}$ Minglong Zhang, ${ }^{\mathrm{a}}$ Tao Huang, ${ }^{\mathrm{a}}$ Shuyun Shi, ${ }^{\mathrm{c}}$ and Changwei Zhang ${ }^{\mathrm{d}}$

The flavor fingerprint of Eucommia ulmoides Oliv. bark (EUb) and its fermentation product were investigated, and volatile compounds were analyzed using headspace gas chromatography-ion mobility spectrometry (HS-GC-IMS) combined with partial least squares discrimination analysis (PLS-DA). A total of 71 peaks were identified, of which 51 target compounds were characterized, including alcohols, aldehydes, acids, and esters. The gallery plot contained 186 signal peaks. The results indicate there were significant differences in the volatile constituents of the three edible fungi. Furthermore, EUb also had its own unique composition of volatile components, and after fermentation with different edible fungi, the volatile components in the product changed significantly compared to the raw materials. A PLS-DA was performed based on the signal intensity of the target volatile compounds obtained; the results clearly showed that the samples (edible fungi, EUb, and different fermentation products) in a relatively independent space were well distinguished. This further illustrated that the composition and content of volatile components from EUb were significantly changed by microorganisms through biofermentation. Combining the signal intensity of the flavor substance, the difference between the different fermentation products was also clearly observed, and the flavor compound's fingerprint was established by HSGC-IMS and PLS-DA methods.
\end{abstract}

Keywords: Ion mobility spectrometry; Eucommia ulmoides Oliv. bark; Characteristic volatile compounds; Fermentation product; Partial least squares discrimination analysis

Contact information: a: Guangdong Provincial Key Laboratory of Emergency Test for Dangerous Chemicals, Guangdong Institute of Analysis, Guangzhou 510070, China; b: School of Chemistry, Sun YatSen University, Guangzhou 510275, China; c: College of Chemistry and Chemical Engineer, Central South University, Changsha 410083, China; d: Institute of Chemical Industry of Forest Products, CAF, Nanjing 210042, China; *Corresponding authors: pengmj163@163.com; cesshzhg@mail.sysu.edu.cn

\section{INTRODUCTION}

Ion mobility spectrometry (IMS) is a quick response, highly sensitive, and portable analytical technique for characterizing chemical substances based on the velocity of gaseous ions in an electrical field at ambient pressure (Holopainen et al. 2013; Tzschoppe et al. 2016). This method offers an alternative to conventionally used methods for both practical application and laboratory analysis. Ion mobility spectrometry is also more easily adapted to real time monitoring than many other analytical instruments (Márquez-Sillero et al. 2011). Operation of IMS does not require complex pre-processing preparation of the sample, and its efficiency has been demonstrated for analysis and characterization of volatile compounds in samples of diverse nature, environmental, and food samples (Garrido-Delgado et al. 2015; Chouinard et al. 2016; Zou et al. 2016; Gerhardt et al. 2017; 
Arroyo-Manzanaresa et al. 2018). At the same time, the ion mobility rate of IMS is only related to the substance itself, it is absolute, and the qualitative analysis is accurate. Therefore, the method is widely used in the detection and identification of flavor substances, drugs, explosives, and chemical agents (Hajialigol et al. 2012; Leonhardt 2013; Fink et al. 2014; Gallegos et al. 2017; Gerhardt et al. 2018; Mochalski et al. 2018; Li et al. 2019).

Although IMS has many advantages for complex samples, especially for complex systems in food and agricultural products, the analysis characteristics are often limited. When IMS is used as a detector to analyze complex samples, there is a problem of cross sensitivity, which mainly includes three cases: (i) Several compounds have highly similar mobility, such that it is difficult to distinguish these compounds; (ii) The ions of some compounds will quench each other in the ionization zone; (iii) When one or several compounds are concentrated, it will affect the formation of other compound ions, thus affecting the analysis and identification (Kanu and Hill 2004; Jafari et al. 2012). In contrast, the most prominent feature of conventional gas chromatography for compound detection is its high separation efficiency, which can analyze almost all volatile compounds. However, the analysis time of gas chromatography is generally measured in minutes, which cannot meet the needs of rapid analysis. Additionally, the retention time of gas chromatography (GC) may change with the change of the stationary phase, and it is difficult to qualitatively analyze the complex unknowns based only on the retained values. The combination of IMS with GC technology will overcome these limitations and obtain satisfactory analysis results. The basic analysis principle of GC-IMS is as follows: complex mixed samples are first separated by GC, then they enter the IMS reaction zone as a single component, and corresponding product ions are formed in the ionization zone. The product ions can enter the migration zone and be separated by two dimensions through ion gate pulse action. The separated product ions are detected after reaching the Faraday disk in turn (Limero et al. 2015; Puton and Namieśnik 2016; Zhang et al. 2016). Therefore, GCIMS technology has a good application prospect in the analysis of volatile components in complex samples.

Taste and aroma are important features of plant resources. Therefore, the study of volatile metabolites can be used to define the sample quality, to characterize the sample, to evaluate changes during the grow process, and also to enable the standardization of sample making with better control of the process (Castro-Muñoz 2019). Availability of new or additional analytical techniques as supporting tools for currently used methods may be helpful to find the difference and characteristic compound from natural plant resources. Natural plant resources are rich in active ingredients and nutrients and have broad prospects for development and utilization. It is worth noting that two-way fermentation (plant matrixfungal two-way solid fermentation engineering) is one of the effective biotechnologies for development and application of natural plant resources. Microorganisms are characterized by a wide variety and strong metabolic capacity. Biotransformation is currently the most promising method for discovering novel compounds (Akacha and Gargouri 2015). Many microorganisms produce new aroma substances when they grow on a plant-derived biobased material as a fermentation substrate. There are four main types of flavor compounds produced by microbial fermentation: pyrazines, lactones, terpenes, and esters (Liese and Filho 1999; Akacha and Gargouri 2015; Vajpeyi and Chandran 2015). The basic principle of the technology is that the medicinal fungus is selected as the fermentation strain, and the medicinal material or the medicinal slag is used as the medicinal matrix to form a common fermentation combination, and under suitable conditions, they are subjected to solid 
fermentation. In this process, the drug matrix provides the nutrients required by the fungus, and is also affected by the enzymes from the fungus to change its own tissues and components, and to produce new chemical ingredients. Meanwhile, the flavor characteristics and appearance of the sample can be significantly improved. The fermentation product is also considered as a development material with high utilization value. Therefore, it is of the utmost research interest (Akacha and Gargouri 2015; BelRhlid et al. 2018).

It is well known that Eucommia ulmoides Oliv. has always been one of the most nourishing herbs in China. Eucommia ulmoides Oliv. bark (EUb) contains many active ingredients, such as lignans, iridoids, flavonoids, phenylpropanoids, and polysaccharides, which have the effects of lowering blood pressure, regulating blood lipids, preventing osteoporosis, lowering blood sugar, calming nerves, and resisting fatigue (He et al. 2014; Hirata et al. 2014; Li et al. 2016; Zhu and Sun 2018). It has a high utilization value and is widely used in food, pharmaceutical, and cosmetic industries. In recent years, the chemical composition, activity, and bioavailability of EUb has continually been the focus of attention (Zhu and Sun 2018), but the composition of volatile components in EUb is often ignored. Moreover, the powerful potential of GC-IMS technology has not been previously adopted for the analysis of EUb, and the investigation of volatile components from fermentation products has rarely been reported. At the same time, the Ganoderma lucidum (GL) strain, Hericium erinaceus (HE) strain, and Griflola frondosa (GF) strain are important edible fungi and have obvious health benefits and medicinal value (Xu et al. 2010; He et al. 2017; Zhao et al. 2017) because the two-way fermentation of natural plants plays an important role in increasing the activity of raw materials and broadening the range of applications. Solid-state fermentation of different edible fungi and EUb may produce some novel compounds. Meanwhile, the separation of GC-IMS is based on the specific drift times that ionized compounds need to pass a fixed distance in a defined electric field. Therefore, this phenomenon is worth exploring, and it is also necessary to analyze the characteristic volatile components of EUb and its fermentation products using the GC-IMS method.

The objective of this study is to develop a quick and simple method to detect volatile substances and investigate the characteristics of volatile compounds from EUb and different edible fungi and fermentation products using the IMS technology. Fingerprints of different samples were established based on the composition of the characteristic compounds detected. Differences between the samples were also analyzed by fingerprints obtained and the partial least squares discrimination analysis (PLS-DA) model. Furthermore, some of the marked compounds were identified throughout the spectrum, and the composition and relative content in different samples were analyzed. These results will provide a reference for an in-depth understanding of the chemical composition of Eucommia ulmoides Oliv. resources and the application of EUb fermentation products.

\section{EXPERIMENTAL}

\section{Materials}

Eucommia ulmoides Oliv. bark was collected from Cili Du-zhong Forestry Centre (Zhangjiajie, China). The fresh bark was dried at $60{ }^{\circ} \mathrm{C}$, and then the sample was pulverized and stored at $4{ }^{\circ} \mathrm{C}$ before use. 
Ganoderma lucidum (GL) preservation strain (strain number GDMCC5.250), Hericium erinaceus (HE) preservation strain (strain number GDMCC5.66), and Griflola frondosa (GF) preservation strain (strain number GDMCC5.63) were purchased from Guangdong Institute of Microbiology Culture Collection (Guangzhou, China).

All of the reagents used in the experiment were of analytical grade. Ultrapure water (18 M $\Omega / \mathrm{cm}$, Milli-Q Plus system; Millipore, Bedford, MA, USA) was used throughout the work. Nitrogen gas with a purity of 5.0 was utilized during the samples analysis process.

\section{Methods}

Solid state fermentation process

The prepared sample of EUb powder was selected, and then an appropriate amount of water was added until the sample was wet. Then the sample was placed in the cultivation bag after being uniformly stirred. These samples were bandaged after being covered with a cotton plug and sterilized for $50 \mathrm{~min}$ at $121^{\circ} \mathrm{C}$. After the sample was cooled to room temperature, under aseptic conditions, the Ganoderma lucidum strain, Hericium erinaceus strain, Griflola frondosa strain, and Ganoderma lucidum-Griflola frondosa complex strain were inoculated into the fermentation medium of the EUb sample. Four fermentation groups were selected for experiments, and cultured in the dark at 24 to $28{ }^{\circ} \mathrm{C}$ until the mycelium was overgrown with the cultivation bag to stop the fermentation. Then, the sample was removed to obtain a different fermented fungus substance. These fermentation products were stored in low temperature conditions until analyzed. There were eight types of samples in the experiment: Ganoderma lucidum microbial strain (GL-M), Hericium erinaceus microbial strain (HE-M), Griflola frondosa microbial strain (GF-M), Eucommia ulmoides Oliv. bark (EUb), Ganoderma lucidum and Eucommia ulmoides Oliv. bark fermentation group (GL-EUb-F), Hericium erinaceus and Eucommia ulmoides Oliv. bark fermentation group (HE-EUb-F), Griflola frondosa and Eucommia ulmoides Oliv. bark fermentation group (GF-EUb-F), and Ganoderma lucidum-Griflola frondosa and Eucommia ulmoides Oliv. bark fermentation group (GL-GF-EUb-F).

\section{Headspace (HS)-GC-IMS analysis}

The GC-IMS instrument used (FlavourSpec ${ }^{\circledR}$; Gesellschaft für Analytische Sensorsysteme $\mathrm{mbH}$, Dortmund, Germany) was equipped with a heated splitless injector, which enabled direct sampling of the headspace from the samples. For better reproducibility, the instrument was coupled to an automatic sampler unit (CTC-PAL; CTC Analytics AG, Zwingen, Switzerland). Samples were analyzed by an IMS (FlavourSpec®; Gesellschaft für Analytische Sensorsysteme $\mathrm{mbH}$, Dortmund, Germany) with a radioactive ionization source $\left(\mathrm{H}^{3}\right)$ for ionization. The $\mathrm{GC}$ was equipped with a non-polar capillary column (FS-SE-54-CB, 94\% methyl, 5\% phenyl, and 1\% vinyl silicone), with $15 \mathrm{~m} \times 0.53$ $\mathrm{mm} \times 0.25 \mu \mathrm{m}$ film thickness. For IMS measurement, different samples were weighed into 20 -mL glass vials that were closed with polypropylene (PP) caps with polytetrafluoroethylene (PTFE)/silicon septa, and was incubated at $80{ }^{\circ} \mathrm{C}$ for $20 \mathrm{~min}$. Then, a headspace volume of $300 \mu \mathrm{L}$ was automatically injected via a heated syringe at $80{ }^{\circ} \mathrm{C}$. The carrier gas (nitrogen gas) ramp was programmed as follows: the initial carrier gas flow was 2.0 $\mathrm{mL} / \mathrm{min}$, which was held for $2 \mathrm{~min}$, then the flow was increased to $20 \mathrm{~mL} / \mathrm{min}$ in $8 \mathrm{~min}$, and the carrier gas flow was ramped up to $100 \mathrm{~mL} / \mathrm{min}$ at $18 \mathrm{~mL} / \mathrm{min}$. Subsequently, the flow rate continued to increase to $150 \mathrm{~mL} / \mathrm{min}$ at a rate of $5 \mathrm{~mL} / \mathrm{min}$. In addition, the carrier gas passed through the GC-IMS injector inserting the sample into the GC column. The samples were eluted using isothermal mode and driven to the ionization chamber. In this 
process, the compounds were ionized by a tritium source at atmospheric pressure. Then, the ions were placed into a drift tube (5- $\mathrm{cm}$ length) through the shutter grid. The flow of drift gas (nitrogen gas) flow was set at $150 \mathrm{~mL} / \mathrm{min}$. Each spectrum was obtained by an average of 32 scans (Tzschoppe et al. 2016).

\section{Data analysis}

Chemometric processing of the ion mobility data was completed with SPSS 19.0 (SPSS, Inc., Chicago, IL, USA) software. The PLS-DA was applied to reduce the dimensionality of the data set retaining the maximum variability present in the original data and eliminating possible dependence between variables. Data analysis of GC-IMS was performed using Laboratory Analytical Viewer (LAV) software (Version 2.000, G.A.S. Gesellschaft für Analytische Sensorsysteme mbH., Dortmund, Germany). An initial exploratory data analysis by PLS-DA was included using the area of different peaks selected from the topographic plot shown in the analysis results. Moreover, the differentiation of the sample by PLS-DA was also carried out using the whole spectral information and using SIMCA-P 14.0 (Umetrics, Umea, Sweden).

The GC-IMS analysis results were shown in a two-dimensional graph in which each analyte is represented by a spot that is characterized by the retention time (measured in seconds, on the y-axis), the drift time (measured in milliseconds, on the x-axis), and the intensity of the signal (Gerhardt et al. 2017; Cavanna et al. 2019).

\section{RESULTS AND DISCUSSION}

\section{Topographic Plot of Different Samples}

IMS is a simple, portable, and sensitive instrumental analytical technique for the detection and monitoring of volatile and semi-volatile compounds present in the natural extract (Gallegos et al. 2015). This technique is based on the gas-phase separation of the resulting ions under a weak electric field at ambient pressure. The migration velocity depends on the mass, size, structure, and spatial distribution of an internal electric charge. The light compact ions travel faster in the drift tube of the instrument and reach the detector before the heavier ions (Vautz et al. 2006). The IMS presents a faster method to determine quality characteristics of foodstuffs and has already been used to determine the separation of various samples via headspace analysis (Gallegos et al. 2015). Taking the analysis results of EUb as an example, a GC-IMS analysis results in a topographic plot as shown in Fig. 1. It is a two-dimensional map in which the y-axis represents the retention time in the chromatographic column (in seconds), the x-axis represents the drift time in the drift tube (in milliseconds), and the color of the signal intensity shows the relative content of the different compounds.

To improve the accuracy of data analysis, further analysis of retention time and relative content was performed by the software LAV to visualize all data analysis results as much as possible. Therefore, data preprocessing is necessary for data analysis and spectrum presentation. The smoothing procedure and baseline correction were used in the preprocessing (Arroyo-Manzanaresa et al. 2018). The second strategy consisted of the selection of individual signals from the topographic plot (Fig. 1) of the EUb samples to conduct the chemometric treatment. 


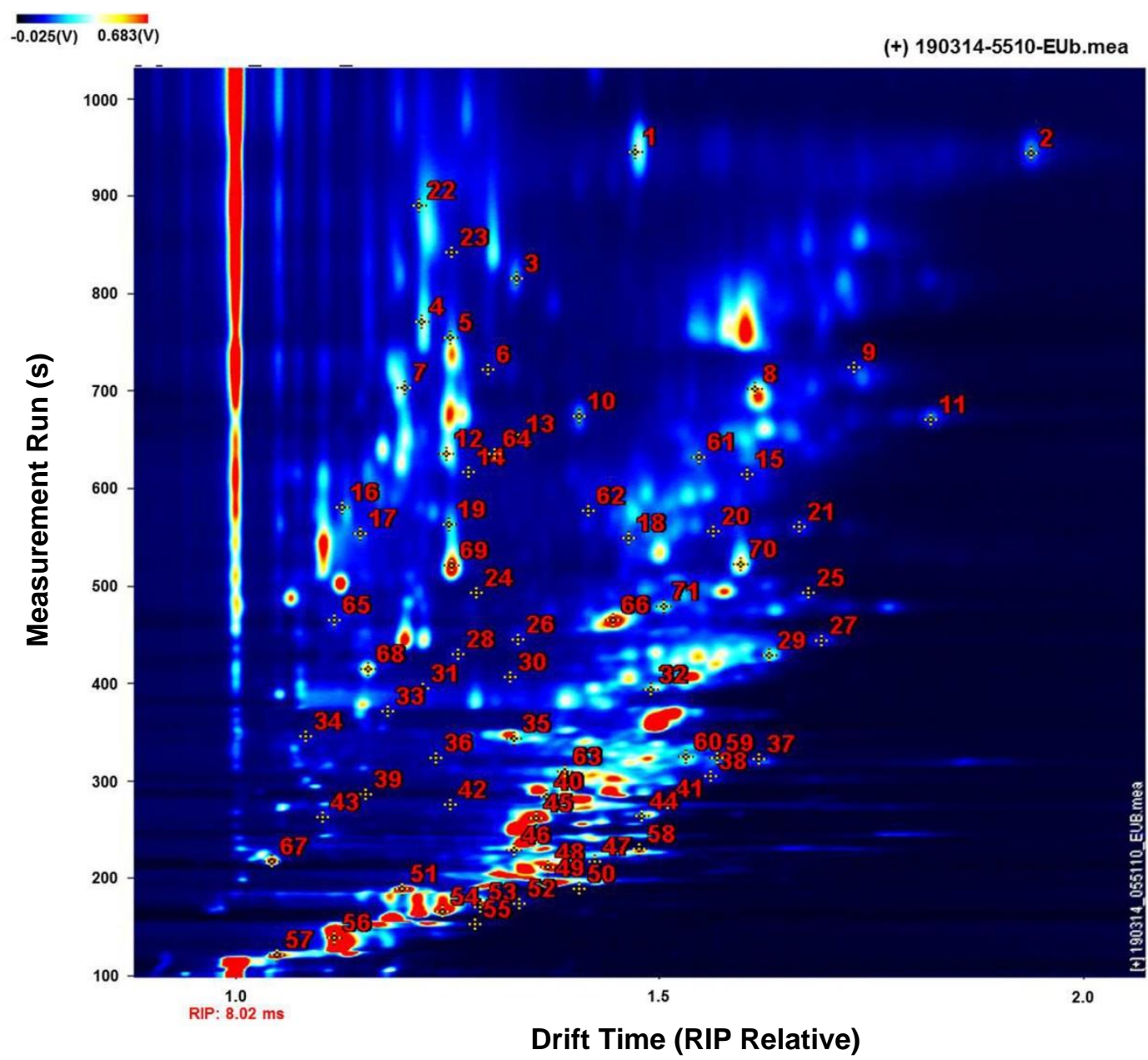

Fig. 1. Imaging of volatile compounds represented by GC-IMS for EUb

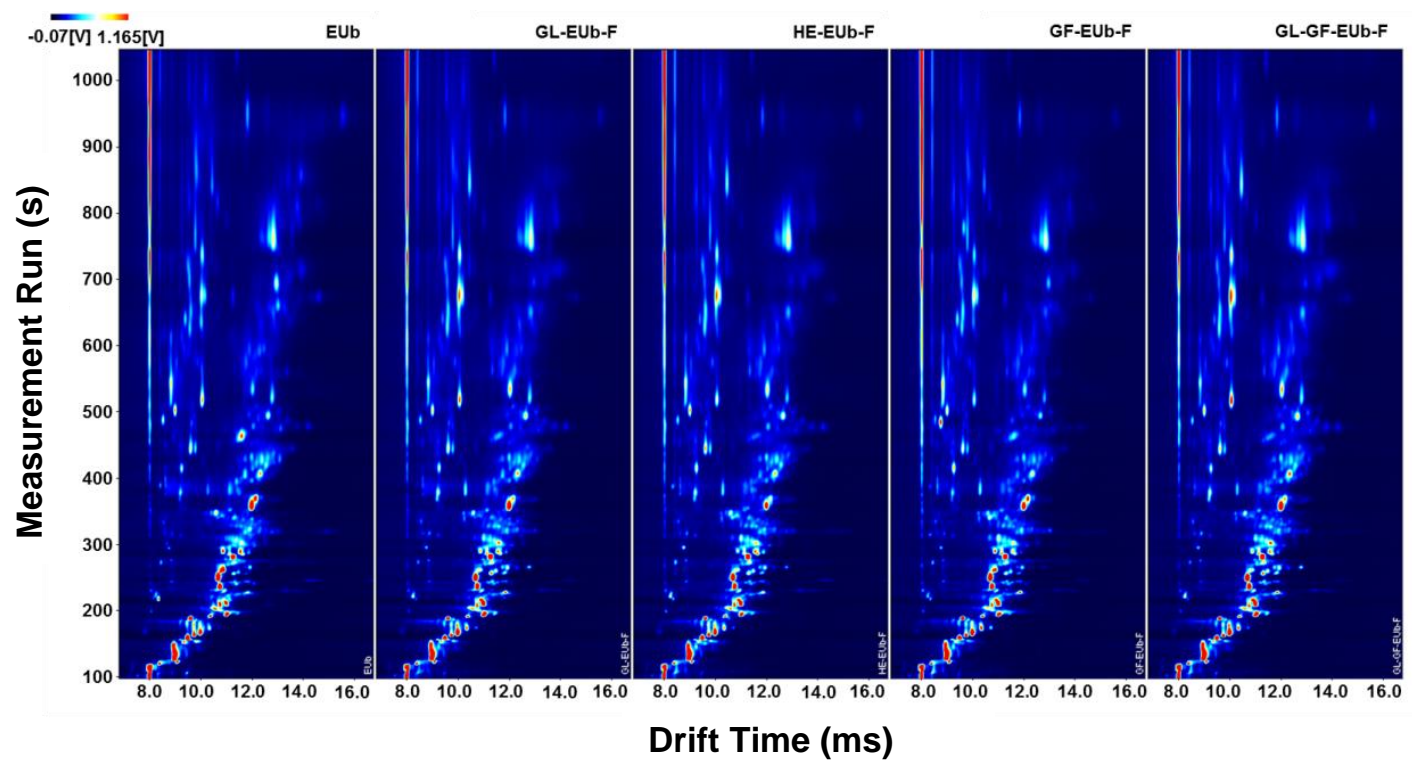

Fig. 2. Comparison of ion migration chromatogram of different samples (edible fungi and Eucommia ulmoides Oliv. bark and its fermentation products) 


\section{A}

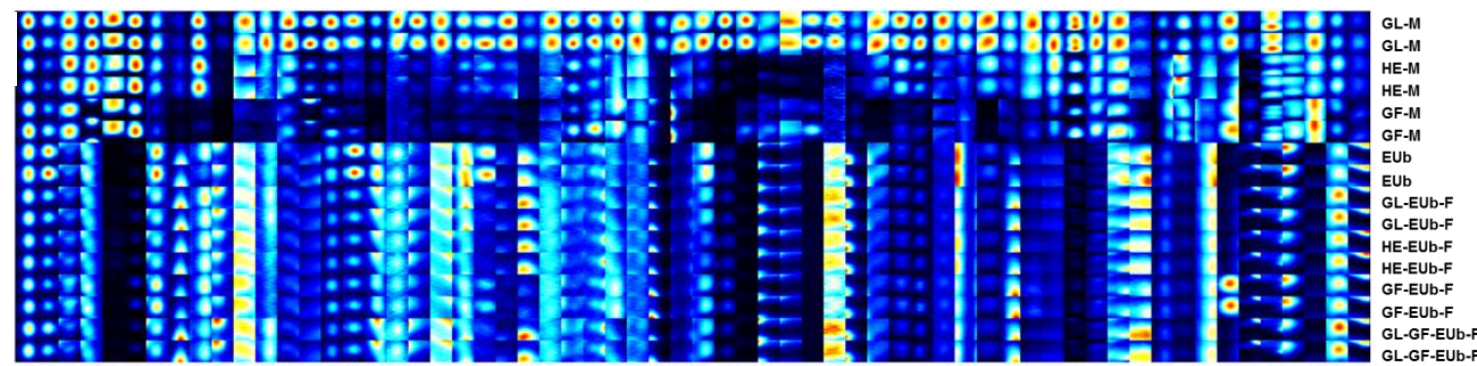

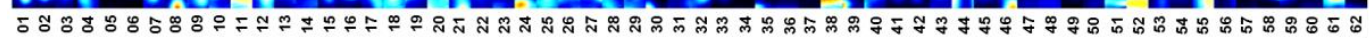

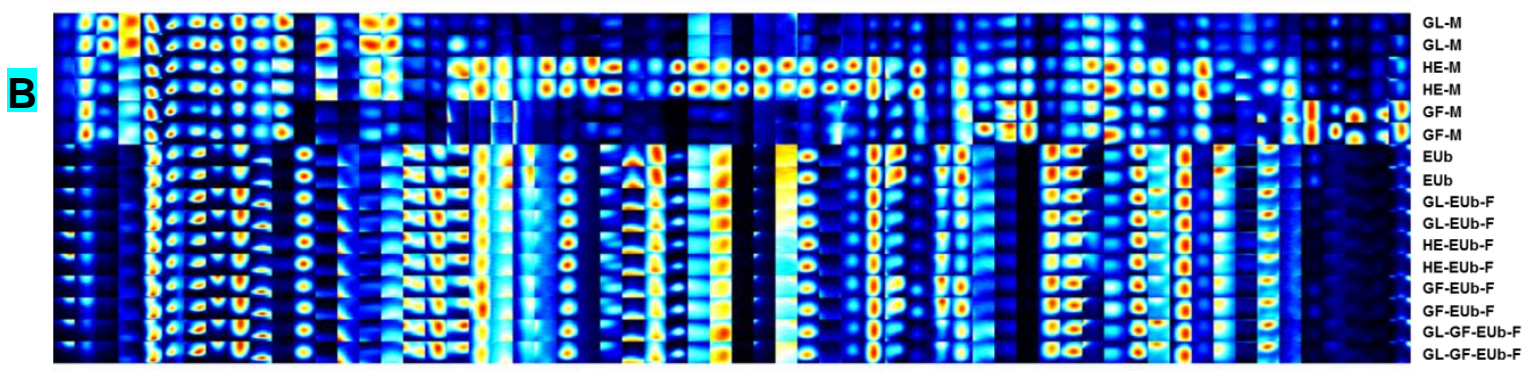

๓

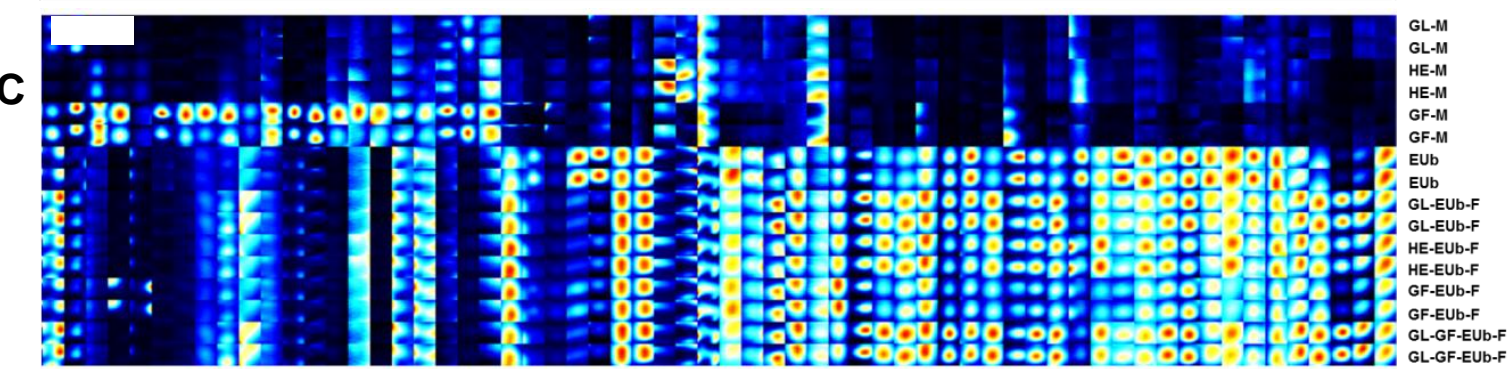

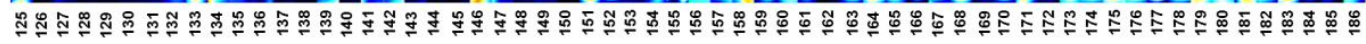

Fig. 3. Gallery plot of the signal peak areas obtained from different sample (edible fungi and Eucommia ulmoides Oliv. bark and its fermentation products)

Based on the results obtained from the data analysis, the position of a signal in the topographical plot is characteristic and can be used to distinguish different compounds and investigate the differences from different samples. The signal intensity represents the relative concentration of the substances (Perl et al. 2011). The comparison of topographic plots obtained for both samples with GC-IMS is also shown in Fig. 2. Although the signal intensity and quantity of different compounds were different, almost all signals were observed between 100 and $800 \mathrm{~s}$ (Arroyo-Manzanaresa et al. 2018). Figure 2 shows that most of the signals appeared between 100 and $600 \mathrm{~s}$ of retention time, although there were some important signals between 700 to $800 \mathrm{~s}$ for EUb and its fermentation product, which was more intense compared with the one observed in other samples. Notice that polar compounds were least retained on the column; therefore, they were concentrated in the first part of the spectrum (between 100 and $300 \mathrm{~s}$ of retention time) with litter resolution between them. In the remaining zones, there were certain maker compounds that were only present in in the corresponding sample, and there were differences in different samples.

In this study, a simpler and quicker GC-IMS technology was proposed for the discrimination of EUb according to their volatile components. It can be seen from Fig. 3 that a total of 186 signal peaks were found from the three edible fungi (GL-M, HE-M, and GF-M) and different EUb samples (EUb, GL-EUb-F, HE-EUb-F, GF-EUb-F, and GL-GFEUb-F) by HS-GC-IMS technology. In terms of three edible fungi, first, some compounds were found in these three samples and the relative content difference of some of them was 
obvious. According to the polarity, boiling point, and retention time, the analysis results of the three strains show their respective composition characteristics in the spectra. As shown in Figs. 2 and 3, the results showed that different edible fungi have their own unique composition of volatile components. In addition to some common compounds, different edible fungi had their own corresponding characteristic areas. At the same time, it also showed that this detection method can be fully applied to find the difference between different samples. In view of the different chemical composition and flavor differences of different edible fungi, applying them to fermentation treatment, some interesting results may be produced and have important research value. Figure 3 also shows that for the 186 signal peaks that were observed, the relative content of some of the three edible fungi was low or even absent. Furthermore, some compounds were found in both three edible fungi and EUb materials, but their relative contents were different according to the analysis results in Fig. 3. From the perspective of the composition of the entire fingerprint, although some compounds could be found in the three edible fungi and EUb, the composition of the overall compound was different. The characteristics of volatile constituents could also be considered as important indicators for identifying the source of raw materials. This also meant that different samples had their own characteristic compounds. These could be applied to the rapid qualitative analysis of samples, and the results could be quickly obtained using HS-GC-IMS technology. Through the biotransformation technology, different edible fungi and EUb were treated by solid state fermentation. This method would have an important impact on the new target components, characteristic volatile components, and flavor improvement of the fermentation products.

\section{Characteristic of volatile compounds from fermentation product}

The results for volatile compounds from samples using GC-IMS are shown in Figs. 2 and 3. The two-dimensional fingerprint is presented as follows: the vertical axis stands for retention time, and the horizontal axis displays the IMS spectrum. The figures showed the proportion of the drift time of the ions in relation to the reaction-ion-peak (RIP). Although the two-dimensional spectrum appears to present complex sample information from the surface, it was easier to perform the evaluation with marked areas. Figures 1 and 2 representatively show the topographic map formed with a colored circle (Wang et al. 2019). Some compounds were identified by the NIST-database and showed corresponding compounds with great probability. This was a molecule that had a strong red character. At the same time, nearly 70 molecules are marked, and a gallery plot was generated by the analysis software. In a gallery plot, the marked molecules are shown in an isolated table form. It is easy to intuitively analyze the changes and differences between different samples in this way. Based on the above results, the gallery plot shows that the samples were similar in many ways. However, differences were also visible. For some compounds, the edible fungi were less complex and less intense at a couple of marked areas in comparison to the different EUb samples. This was expressed by a lower peak height, smaller peak contour, and color code. There were marked molecules that appear as top note.

Fermentation products can be obtained by solid-state fermentation of GL, HE, GF, and GL-GF with EUb. The volatile components of the each were analyzed by the HS-GCIMS method. The results are displayed in Figs. 2 and 3. The composition and relative content of the volatile compounds in the corresponding fermentation products were all changed compared to the raw materials of EUb. The topographic plots showed that the relative contents of some compounds in edible fungi were reduced or even disappeared through fermentation technology. The corresponding change was marked in the Figs. 2 and 
3. The EUb also had similar trends, but in terms of quantity of compounds, it was far less than edible fungi. Meanwhile, different novel compounds were produced in the fermentation products based on the different edible fungi, and the composition and relative content of the compounds in the final fermentation product were also different. Compared with the raw material of EUb, after the solid fermentation of the three edible fungi, the relative content of 55 compounds increased, and the relative content of 19 compounds was similar or slightly increased. At the same time, the relative content of 48 compounds was reduced after fermentation treatment, and another 21 compounds were close to the raw material with a slight decrease. It is worth noting that there were 12 compounds that were not found in the product after fermentation by different edible fungi. In addition, after the solid fermentation treatment, the relative content of 31 compounds also changed (increased or decreased) based on the difference of fermentation strains, and the extent of change was also obvious. This also indicated that different edible fungi could change the composition characteristics of volatile components in EUb through solid state fermentation. Choosing the suitable edible fungus, sample and fermentation conditions were of great significance for discovering new target compounds and improving the original flavor of the raw materials. Furthermore, although the relative content of most of the compounds in the fermentation product changed after fermentation treatment, compared with the EUb, and the changes of some compounds were obvious, the basic composition of the whole volatile compound was similar to the raw materials, despite some new compounds produced and the disappearing compounds. However, compared with the three edible fungi, the volatile components from the fermentation product were changed. It can be seen from Fig. 3 that a large number of compounds were reduced or disappeared, which further indicated that during the fermentation process, microorganisms affect their own flavor components through their own metabolism, and the fermentation substrate also played an important role in the fermentation process. Any substance has its own unique volatile substances. Edible fungi and EUb contained their own different characteristic flavor compounds, but after biological fermentation, some interesting results were observed. There was obvious adjustment and improvement on the flavor of the raw materials. The experimental results showed that the IMS technology could efficiently and quickly detect and analyze volatile components in samples. First, in the process of solid fermentation through a specific fermentation substrate, the flavor composition of different microorganisms was changed. Second, by using microbial metabolism, the shape and flavor of the raw material matrix of EUb was also changed. Therefore, the selection of suitable microbial strains and fermentation conditions could effectively improve the appearance, functional ingredients, nutritional value, taste, and flavor of the raw material samples.

\section{Determination of volatile compounds identified from samples}

To identify the selected markers, a commercial GC-IMS Library was used, and the compounds tentatively identified using this library were confirmed by comparison of retention time and drift time (Gallegos et al. 2017). As shown in Fig. 1, all the selected compounds were marked in the spectrum. Table 1 showed the data for the selected markers identified by their corresponding retention time, drift time, and reactant ion peak. Differences were evident in terms of signal intensities; in particular, different samples contain high concentrations of characteristic compounds. This fact was also observed from other previous reports (Arroyo-Manzanaresa et al. 2018). Different samples were analyzed using the optimized GC-IMS method. In Fig. 3, the characteristic topographic plots obtained for different samples is shown. As observed, different fingerprints were obtained. 
The compounds that have been identified mainly include alcohols, aldehydes, ketones, acids, esters, and so on (Gallegos et al. 2015). As shown in Fig. 3, both samples examined gave different profiles of volatile compounds by GC-IMS. Some volatile compounds were identified as characteristic for EUb (identified compounds) as well as unknown compounds. For other samples, some compounds were also found to be characteristic by this method, but these compounds need to be further identified.

Table 1. GC-IMS Integration Parameters of Volatile Compounds Identified to Distinguish Different Samples

\begin{tabular}{|c|c|c|c|c|c|c|c|c|}
\hline$\#$ & $\begin{array}{c}\text { Gallery } \\
\text { Plot-area } \\
\text { Label }\end{array}$ & Compound & Formula & MW & $\mathrm{RI}$ & $\mathrm{Rt}(\mathrm{s})$ & $\begin{array}{c}\text { Dt (RIP } \\
\text { Relative) }\end{array}$ & Comment \\
\hline 1 & 1 & Nonanal & $\mathrm{C}_{9} \mathrm{H}_{18} \mathrm{O}$ & 142.2 & 1105.9 & 945.36 & 1.474 & Monomer \\
\hline 2 & 2 & Nonanal & $\mathrm{C}_{9} \mathrm{H}_{18} \mathrm{O}$ & 142.2 & 1105.1 & 943.02 & 1.941 & Dimer \\
\hline 3 & 7 & (E)-2-Octenal & $\mathrm{C}_{8} \mathrm{H}_{14} \mathrm{O}$ & 126.2 & 1059.3 & 815.1 & 1.333 & \\
\hline 4 & 82 & Limonene & $\mathrm{C}_{10} \mathrm{H}_{16}$ & 136.2 & 1043.2 & 771.03 & 1.222 & \\
\hline 5 & 8 & $\begin{array}{l}\text { Phenylacet- } \\
\text { aldehyde }\end{array}$ & $\mathrm{C}_{8} \mathrm{H}_{8} \mathrm{O}$ & 120.2 & 1037.1 & 754.65 & 1.255 & \\
\hline 6 & 9 & 1,8-Cineol & $\mathrm{C}_{10} \mathrm{H}_{18} \mathrm{O}$ & 154.3 & 1024.9 & 721.89 & 1.3 & Monomer \\
\hline 7 & 4 & $\begin{array}{l}\text { ( E, E)-2,4- } \\
\text { Heptadienal }\end{array}$ & $\mathrm{C}_{7} \mathrm{H}_{10} \mathrm{O}$ & 110.2 & 1017.5 & 702.39 & 1.201 & Monomer \\
\hline 8 & 120 & $\begin{array}{l}\text { ( E, E)-2,4- } \\
\text { Heptadienal }\end{array}$ & $\mathrm{C}_{7} \mathrm{H}_{10} \mathrm{O}$ & 110.2 & 1017.2 & 701.61 & 1.615 & Dimer \\
\hline 9 & 52 & 1,8-Cineol & $\mathrm{C}_{10} \mathrm{H}_{18} \mathrm{O}$ & 154.3 & 1025.5 & 723.45 & 1.732 & Dimer \\
\hline 10 & 15 & Octanal & $\mathrm{C}_{10} \mathrm{H}_{18} \mathrm{O}$ & 154.3 & 1006.3 & 673.53 & 1.408 & Monomer \\
\hline 11 & 16 & Octanal & $\mathrm{C}_{10} \mathrm{H}_{18} \mathrm{O}$ & 154.3 & 1005.1 & 670.41 & 1.822 & Dimer \\
\hline 12 & 17 & 2-Pentylfuran & $\mathrm{C}_{9} \mathrm{H}_{14} \mathrm{O}$ & 138.2 & 990.7 & 634.53 & 1.251 & Monomer \\
\hline 13 & 18 & 2-Octanone & $\mathrm{C}_{8} \mathrm{H}_{16} \mathrm{O}$ & 128.2 & 997.3 & 650.91 & 1.335 & \\
\hline 14 & 19 & 1-Octen-3-one & $\mathrm{C}_{8} \mathrm{H}_{14} \mathrm{O}$ & 126.2 & 983.4 & 616.59 & 1.277 & \\
\hline 15 & 20 & 1-Octen-3-ol & $\mathrm{C}_{8} \mathrm{H}_{16} \mathrm{O}$ & 128.2 & 982.1 & 613.47 & 1.606 & \\
\hline 16 & 53 & $\begin{array}{l}\text { 5-Methyl-2- } \\
\text { furfural }\end{array}$ & $\mathrm{C}_{6} \mathrm{H}_{6} \mathrm{O}_{2}$ & 110.1 & 967.8 & 579.93 & 1.128 & Monomer \\
\hline 17 & 54 & Benzaldehyde & $\mathrm{C}_{7} \mathrm{H}_{6} \mathrm{O}$ & 106.1 & 956.1 & 553.41 & 1.149 & Monomer \\
\hline 18 & 121 & Benzaldehyde & $\mathrm{C}_{7} \mathrm{H}_{6} \mathrm{O}$ & 106.1 & 954 & 548.73 & 1.466 & Dimer \\
\hline 19 & 21 & $\begin{array}{l}\text { 2-Furanmeth- } \\
\text { anol, 5-methyl }\end{array}$ & $\mathrm{C}_{6} \mathrm{H}_{8} \mathrm{O}_{2}$ & 112.1 & 960.3 & 562.77 & 1.254 & Monomer \\
\hline 20 & 26 & $\begin{array}{l}\text { 2-Furanmeth- } \\
\text { nol, 5-methyl }\end{array}$ & $\mathrm{C}_{6} \mathrm{H}_{8} \mathrm{O}_{2}$ & 112.1 & 957.2 & 555.75 & 1.566 & Dimer \\
\hline 21 & 22 & (E)-2-Heptenal & $\mathrm{C}_{7} \mathrm{H}_{12} \mathrm{O}$ & 112.2 & 959.2 & 560.43 & 1.667 & \\
\hline 22 & 55 & Linalool & $\mathrm{C}_{10} \mathrm{H}_{18} \mathrm{O}$ & 154.3 & 1086 & 889.59 & 1.218 & \\
\hline 23 & 85 & Linalool oxide & $\begin{array}{c}\mathrm{C}_{10} \mathrm{H}_{18} \mathrm{O} \\
2\end{array}$ & 170.3 & 1069 & 842.20 & 1.257 & \\
\hline 24 & 23 & $\begin{array}{c}\text { Methyl } \\
\text { hexanoate }\end{array}$ & $\mathrm{C}_{7} \mathrm{H}_{14} \mathrm{O}_{2}$ & 130.2 & 927.2 & 492.96 & 1.286 & Monomer \\
\hline 25 & 24 & $\begin{array}{c}\text { Methyl } \\
\text { hexanoate }\end{array}$ & $\mathrm{C}_{7} \mathrm{H}_{14} \mathrm{O}_{2}$ & 130.2 & 926.8 & 492.18 & 1.677 & Dimer \\
\hline 26 & 41 & Heptanal & $\mathrm{C}_{7} \mathrm{H}_{14} \mathrm{O}$ & 114.2 & 900.9 & 444.60 & 1.335 & Monomer \\
\hline
\end{tabular}




\begin{tabular}{|c|c|c|c|c|c|c|c|c|}
\hline 27 & 45 & Heptanal & $\mathrm{C}_{7} \mathrm{H}_{14} \mathrm{O}$ & 114.2 & 900 & 443.04 & 1.693 & Dimer \\
\hline 28 & 42 & 2-Heptanone & $\mathrm{C}_{7} \mathrm{H}_{14} \mathrm{O}$ & 114.2 & 892.1 & 429.78 & 1.265 & Monomer \\
\hline 29 & 32 & 2-Heptanone & $\mathrm{C}_{7} \mathrm{H}_{14} \mathrm{O}$ & 114.2 & 891.1 & 428.22 & 1.631 & Dimer \\
\hline 30 & 47 & 1-Hexanol & $\mathrm{C}_{6} \mathrm{H}_{14} \mathrm{O}$ & 102.2 & 876.8 & 405.99 & 1.326 & \\
\hline 31 & 48 & $\begin{array}{l}\text { 3-Methylbut- } \\
\text { anoic acid }\end{array}$ & $\mathrm{C}_{5} \mathrm{H}_{10} \mathrm{O}_{2}$ & 102.1 & 868.7 & 394.29 & 1.223 & Monomer \\
\hline 32 & 81 & $\begin{array}{c}\text { 3- } \\
\text { Methylbutanoi } \\
\text { c acid }\end{array}$ & $\mathrm{C}_{5} \mathrm{H}_{10} \mathrm{O}_{2}$ & 102.1 & 867.6 & 392.73 & 1.492 & Dimer \\
\hline 33 & 3 & 2-Hexen-1-ol & $\mathrm{C}_{6} \mathrm{H}_{12} \mathrm{O}$ & 100.2 & 851.8 & 371.28 & 1.181 & \\
\hline 34 & 123 & 2-Furfural & $\mathrm{C}_{5} \mathrm{H}_{4} \mathrm{O}_{2}$ & 96.1 & 831.1 & 345.54 & 1.084 & Monomer \\
\hline 35 & 88 & 2-Furfural & $\mathrm{C}_{5} \mathrm{H}_{4} \mathrm{O}_{2}$ & 96.1 & 828.8 & 342.81 & 1.33 & Dimer \\
\hline 36 & 43 & Butyl acetate & $\mathrm{C}_{6} \mathrm{H}_{12} \mathrm{O}_{2}$ & 116.2 & 811.1 & 322.72 & 1.239 & Monomer \\
\hline 37 & 33 & Butyl acetate & $\mathrm{C}_{6} \mathrm{H}_{12} \mathrm{O}_{2}$ & 116.2 & 810.1 & 321.56 & 1.62 & Dimer \\
\hline 38 & 49 & Hexanal & $\mathrm{C}_{6} \mathrm{H}_{12} \mathrm{O}$ & 100.2 & 793.6 & 303.81 & 1.562 & \\
\hline 39 & 60 & $\begin{array}{l}\text { 2-Methylpro- } \\
\text { panoic acid }\end{array}$ & $\mathrm{C}_{4} \mathrm{H}_{8} \mathrm{O}_{2}$ & 88.1 & 775.4 & 285.09 & 1.156 & Monomer \\
\hline 40 & 124 & $\begin{array}{l}\text { 2-Methylpro- } \\
\text { panoic acid }\end{array}$ & $\mathrm{C}_{4} \mathrm{H}_{8} \mathrm{O}_{2}$ & 88.1 & 773.1 & 282.75 & 1.369 & Dimer \\
\hline 41 & 77 & 1-Pentanol & $\mathrm{C}_{5} \mathrm{H}_{12} \mathrm{O}$ & 88.1 & 766.7 & 276.32 & 1.512 & Dimer \\
\hline 42 & 50 & 1-Pentanol & $\mathrm{C}_{5} \mathrm{H}_{12} \mathrm{O}$ & 88.1 & 764.9 & 274.56 & 1.255 & Monomer \\
\hline 43 & 64 & $\begin{array}{l}\text { Propanoic } \\
\text { acid }\end{array}$ & $\mathrm{C}_{3} \mathrm{H}_{6} \mathrm{O}_{2}$ & 74.1 & 751.6 & 261.69 & 1.105 & Monomer \\
\hline 44 & 125 & $\begin{array}{l}\text { 2-Methyl-1- } \\
\text { butanol }\end{array}$ & $\mathrm{C}_{5} \mathrm{H}_{12} \mathrm{O}$ & 88.1 & 753.7 & 263.64 & 1.481 & \\
\hline 45 & 101 & $\begin{array}{c}\text { Propanoic } \\
\text { acid }\end{array}$ & $\mathrm{C}_{3} \mathrm{H}_{6} \mathrm{O}_{2}$ & 74.1 & 750.8 & 260.91 & 1.356 & Dimer \\
\hline 46 & 57 & Acetoin & $\mathrm{C}_{4} \mathrm{H}_{8} \mathrm{O}_{2}$ & 88.1 & 713.1 & 228.35 & 1.33 & \\
\hline 47 & 65 & Pentanal & $\mathrm{C}_{5} \mathrm{H}_{10} \mathrm{O}$ & 86.1 & 696.3 & 216.06 & 1.425 & \\
\hline 48 & 80 & 2-Pentanone & $\mathrm{C}_{5} \mathrm{H}_{10} \mathrm{O}$ & 86.1 & 688 & 210.60 & 1.37 & \\
\hline 49 & 69 & 1-Butanol & $\mathrm{C}_{4} \mathrm{H}_{10} \mathrm{O}$ & 74.1 & 658.4 & 194.22 & 1.371 & \\
\hline 50 & 5 & $\begin{array}{l}\text { 3-Methyl- } \\
\text { butanal }\end{array}$ & $\mathrm{C}_{5} \mathrm{H}_{10} \mathrm{O}$ & 86.1 & 645.9 & 188.37 & 1.408 & Dimer \\
\hline 51 & 108 & $\begin{array}{l}\text { 3-Methyl- } \\
\text { butanal }\end{array}$ & $\mathrm{C}_{5} \mathrm{H}_{10} \mathrm{O}$ & 86.1 & 645.9 & 188.37 & 1.198 & Monomer \\
\hline 52 & 6 & Ethyl Acetate & $\mathrm{C}_{4} \mathrm{H}_{8} \mathrm{O}_{2}$ & 88.1 & 608.9 & 173.36 & 1.337 & \\
\hline 53 & 70 & Butanal & $\mathrm{C}_{4} \mathrm{H}_{8} \mathrm{O}$ & 72.1 & 598.9 & 169.65 & 1.291 & \\
\hline 54 & 71 & 2-Butanone & $\mathrm{C}_{4} \mathrm{H}_{8} \mathrm{O}$ & 72.1 & 585.6 & 164.78 & 1.246 & \\
\hline 55 & 73 & $\begin{array}{l}\text { Methyl- } \\
\text { propanal }\end{array}$ & $\mathrm{C}_{4} \mathrm{H}_{8} \mathrm{O}$ & 72.1 & 552.7 & 152.68 & 1.285 & \\
\hline 56 & 67 & Acetone & $\mathrm{C}_{3} \mathrm{H}_{6} \mathrm{O}$ & 58.1 & 512.3 & 137.86 & 1.119 & \\
\hline 57 & 68 & Ethanol & $\mathrm{C}_{2} \mathrm{H}_{6} \mathrm{O}$ & 46.1 & 464.5 & 120.31 & 1.051 & \\
\hline 58 & 91 & Propyl acetate & $\mathrm{C}_{5} \mathrm{H}_{10} \mathrm{O}_{2}$ & 102.1 & 714.1 & 229.12 & 1.478 & \\
\hline 59 & 92 & 2-Hexanol & $\mathrm{C}_{6} \mathrm{H}_{14} \mathrm{O}$ & 102.2 & 811.1 & 322.72 & 1.57 & \\
\hline 60 & 93 & $\begin{array}{l}\text { Ethyl 2-hydro- } \\
\text { xypropanoate }\end{array}$ & $\mathrm{C}_{5} \mathrm{H}_{10} \mathrm{O}_{3}$ & 118.1 & 812.6 & 324.28 & 1.534 & \\
\hline 61 & 132 & 2-Pentylfuran & $\mathrm{C}_{9} \mathrm{H}_{14} \mathrm{O}$ & 138.2 & 989.6 & 631.8 & 1.549 & Dimer \\
\hline
\end{tabular}




\begin{tabular}{|c|c|c|c|c|c|c|c|c|}
\hline 62 & 133 & $\begin{array}{c}\text { 5-Methyl-2- } \\
\text { furfural }\end{array}$ & $\mathrm{C}_{6} \mathrm{H}_{6} \mathrm{O}_{2}$ & 110.1 & 966.2 & 576.03 & 1.418 & Dimer \\
\hline 63 & 142 & Butanoic acid & $\mathrm{C}_{4} \mathrm{H}_{8} \mathrm{O}_{2}$ & 88.1 & 798 & 308.49 & 1.391 & \\
\hline 64 & 38 & 3-Octanone & $\mathrm{C}_{8} \mathrm{H}_{16} \mathrm{O}$ & 128.2 & 990.7 & 634.53 & 1.307 & \\
\hline 65 & 148 & 2-Acetylfuran & $\mathrm{C}_{6} \mathrm{H}_{6} \mathrm{O}_{2}$ & 110.1 & 912 & 464.10 & 1.118 & Monomer \\
\hline 66 & 149 & 2-Acetylfuran & $\mathrm{C}_{6} \mathrm{H}_{6} \mathrm{O}_{2}$ & 110.1 & 912.2 & 464.49 & 1.448 & Dimer \\
\hline 67 & 150 & $\begin{array}{c}\text { 1-Propene-3- } \\
\text { methylthio }\end{array}$ & $\mathrm{C}_{4} \mathrm{H}_{8} \mathrm{~S}$ & 88.2 & 697.4 & 216.84 & 1.045 & \\
\hline 68 & 161 & $\begin{array}{c}\text { Propylsulfide } \\
\mathrm{C}_{6} \mathrm{H}_{14} \mathrm{~S}\end{array}$ & 118.2 & 881.9 & 413.59 & 1.159 & \\
\hline 69 & 166 & $\begin{array}{c}\text { 3-Methyl } \\
\text { valeric acid }\end{array}$ & $\mathrm{C}_{6} \mathrm{H}_{12} \mathrm{O}_{2}$ & 116.2 & 940.6 & 520.26 & 1.256 & Monomer \\
\hline 70 & 167 & $\begin{array}{c}3-M e t h y l \\
\text { valeric acid }\end{array}$ & $\mathrm{C}_{6} \mathrm{H}_{12} \mathrm{O}_{2}$ & 116.2 & 941.4 & 521.82 & 1.598 & Dimer \\
\hline 71 & 173 & Pentanoic acid & $\mathrm{C}_{5} \mathrm{H}_{10} \mathrm{O}_{2}$ & 102.1 & 919.8 & 478.72 & 1.507 & \\
\hline
\end{tabular}

The different samples from edible fungi, Eucommia ulmoides Oliv. bark and its fermentation product were analyzed by HS-GC-IMS in duplicate. The results are shown in the Fig. 2. A topographic plot of each sample is shown as an example of the valuable information (signal intensity, retention time, and drift time) obtained after each individual analysis. Similar spectral fingerprints were obtained for different samples analyzed. According to the intensity of their signals, characteristic compounds in the sample were presented, and some compounds were identified in calculating ion mobility of each sample, and the corresponding data are included in Table 1. Other samples were also determined under the same conditions. The volatile compounds detected from EUb and its fermentation products showed different signal intensities in each sample. Moreover, the authors observed that the samples appeared as proton-bound monomers and dimers in GC-IMS plots. Detailed results are presented in Figs. 2 and 3 and Table 1. It is worth noting that in some cases, some compounds appeared in a region along with other signals, so it was difficult to clearly identify these signals, and for this reason these data were not included in Table 1. These results are similar to previous reports (Borsdorf and Eiceman 2006; Gallegos et al. 2017).

Using IMS analysis techniques, a total of 51 compounds were identified based on migration time, retention index, and corresponding database. The most abundant species were aldehyde compounds, a total of 22 species, followed by 13 alcohol compounds. The number of identified ketones and esters was 9 and 7, respectively. Additionally, 10 acid compounds were found, as well as 10 other classes of compounds included in the identified compounds. In terms of compounds identified after the fermentation, the relative content of ethyl acetate, 1-octen-3-one, 1-octen-3-ol, butyl acetate, 2-heptanone, butyl acetate, 1pentanol, 5-methyl-2-furfural, acetoin, acetone, butanal, 2-butanone, 1-pentanol, 2pentanone, propyl acetate, 2-hexanol, benzaldehyde, 2-pentylfuran, 5-methyl-2-furfural, butanoic acid, and propylsulfide increased to different degrees. Compared to the other groups, nonanal, (E)-2-octenal, octanal, (E)-2-heptenal, 1, 8-cineol, linalool, propanoic acid, (E, E)-2, 4-heptadienal, 2-acetylfuran, 1-propene-3-methylthio, and 3-methyl valeric acid had the highest content in the raw materials, and the relative content was lowered after the fermentation. Additionally, 3-methylbutanal should be considered as a characteristic component of edible fungi and was not detected in EUb and fermentation products. 2Furfural was prominent in the GF group. The signal intensity of phenylacetaldehyde, 2pentylfuran, methyl hexanoate, acetoin, 1-butanol, and 3-methyl valeric acid was the most 
obvious in the GL-GF composite fermentation group. For compounds 2-heptanone and 2methylpropanoic acid, the content of the raw materials was higher than that of the fermentation group. The edible fungus HE reduced the content of 3-methylbutanal in EUb. In the HE fermentation group, the content of pentanoic acid was the highest, and the content of butanoic acid was also higher than other groups. The edible fungus GF did not change the content of the 2-methyl-1-butanol in the raw material, and the other two bacteria and the compound strain reduced the compound content, and the relative content of propylsulfide in the GF fermentation group was the highest. The fermentation of GL and GL-GF increased the signal intensity of 2-pentanone, and the relative content of 1-butanol was improved by GF fermentation. It is worth noting that all edible fungi significantly increased the relative content of 2-butanone by fermentation in the experiment. Through comparison, it was found that the peak intensity of the compounds in different fermentation groups changed to varying degrees after fermentation by the corresponding edible fungi. This also showed that the selection of suitable strains will improve the flavor components of the raw materials through the fermentation process. Meanwhile, some new compounds may also be discovered. Based on the analysis of the compounds that have been identified, there were differences in the composition and relative content of flavors from different species. As shown in Fig. 3, in the respective flavor compound composition regions, the signal intensity of most compounds of edible fungi was higher than that of raw materials and fermentation products, but from the perspective of compound composition, the volatile components in EUb were more abundant. Moreover, considerable results were obtained by HS-GC-IMS technical analysis, and some compound compositions were also consistent with previous literature reports. These results indicated that GC-IMS is suitable for the detection of volatile components in EUb and its fermentation products, and the morphology and content of volatile components of EUb. The application of this method is of great significance for the development of new and promising raw materials using Eucommia ulmoides resources.

Volatile organic compounds were determined with GC-IMS. Further investigations are needed to optimize the GC-IMS setting and in this context, detection limits must be defined. In this study, not all molecules were identified due to database limitations, so in future work, the composition and identification of volatile compounds needs to be further supplemented and improved in combination with other new analytical techniques.

\section{Discrimination of sample by partial least squares discrimination analysis}

Partial least squares discriminant analysis (PLS-DA) is a calculation method of multivariate correction method PLS, which can be used for supervised classification and discriminant analysis. And PLS-DA is the most widely used multivariate data analysis technology in chemometrics. As a supervised dimensionality reduction algorithm, PLSDA based on the difference analysis of the obtained data, more effectively find the maximum interval between each class. This will help to better distinguish samples and categorize analysis (Toraman et al. 2018; Allen et al. 2019). In this work, a PLS-DA classification model was proposed to distinguish the differences in volatile components in EUb and their fermentation products based on GC-IMS signals associated to the type of flavor substances. This describes the systemic patterns of variation between the samples. A plot of PLS-DA where t[2] vs t[1] is represented and the corresponding sample name has been marked. In Fig. 4, the different clusters formed have been discriminated for sample types from raw materials and different fermentation products. 


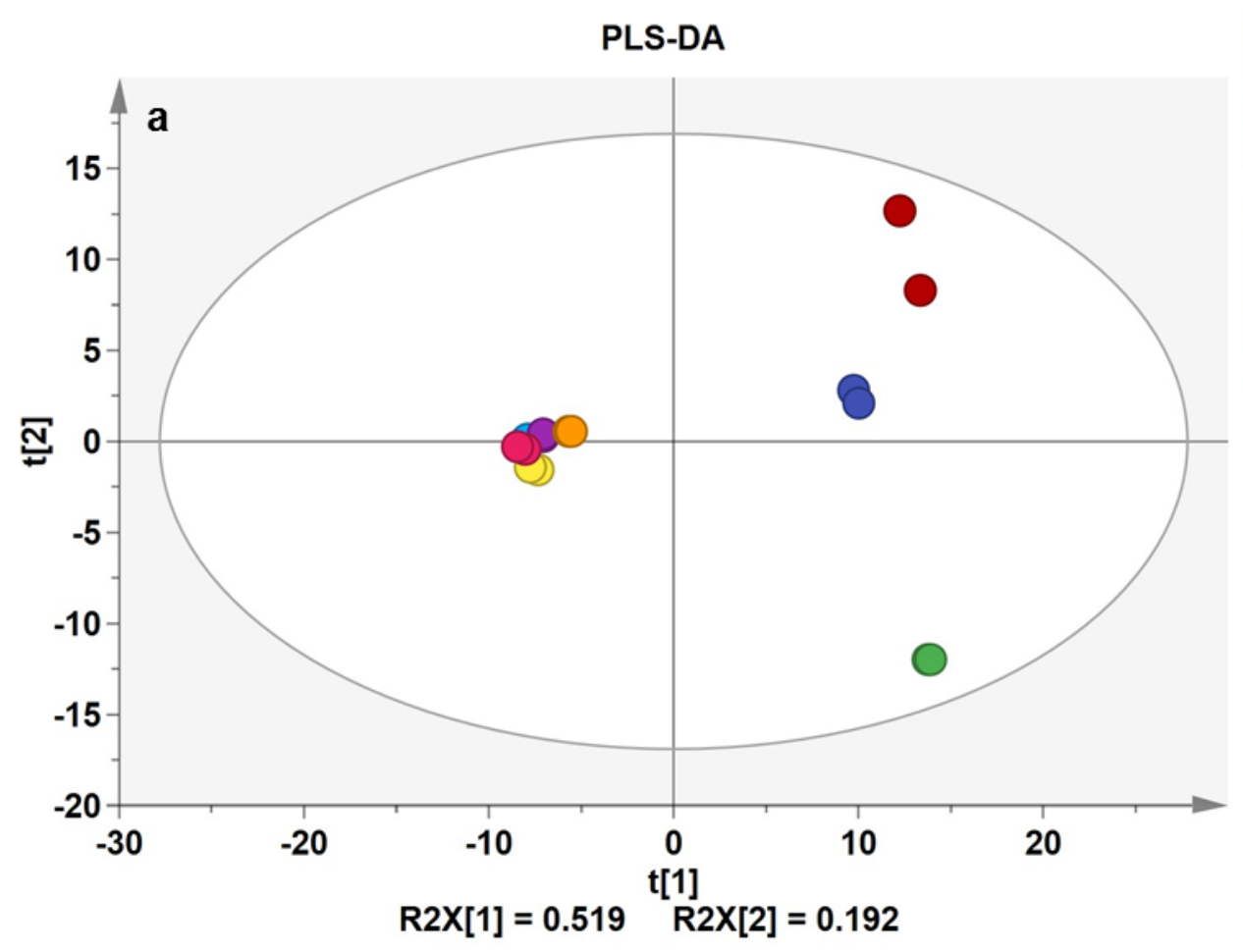

GL-M
HE-M
GF-M
EUb

GL-EUb-F HE-EUb-F GF-EUb-F GL-GF-EL

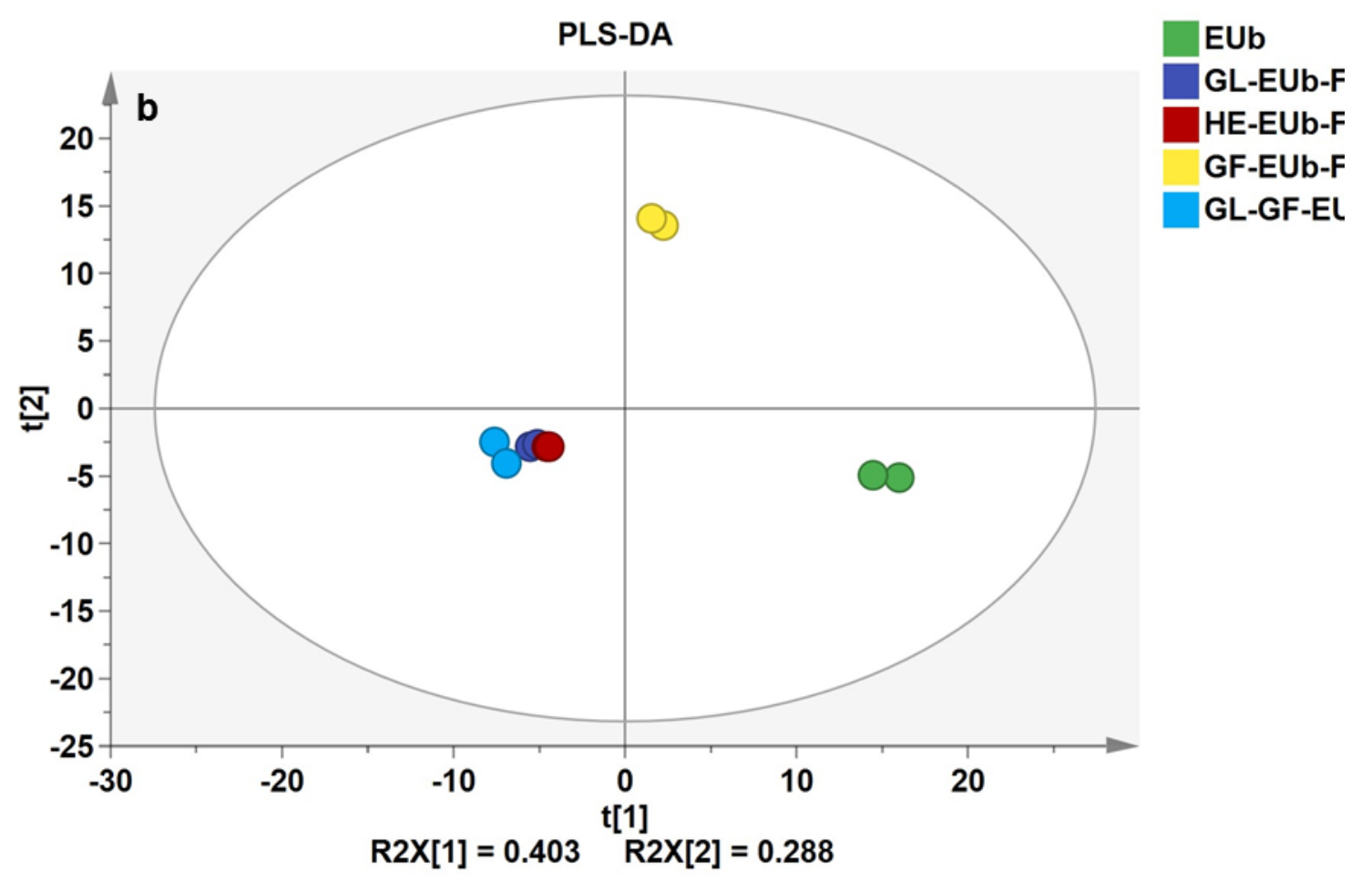

Fig. 4. Partial least squares discrimination analysis based on the signal intensity obtained of volatile compounds from different samples (edible fungi and Eucommia ulmoides Oliv. bark and its fermentation products): $a$ : all the samples of raw materials and fermentation product; $b$ : EUb sample and its different fermentation products, except for edible fungi

The potential of the HS-GC-IMS method to identify different EUb samples could be highlighted using a global analysis (including all data in the region of drift time from 8 
to $14 \mathrm{~ms}$ and a retention time from 100 to $800 \mathrm{~s}$ ) from the topographic plot of each sample (Fig. 2). The data set used to make the PLS-DA was obtained from the GC-IMS analysis. The data used to build the PLS-DA were normalized with respect to the reactant ion peak area. In this study, to further explore the differences in volatile components, GC-IMS signal peak data obtained from different groups of samples (with or without three edible fungi) were analyzed by the PLS-DA model.

According to Fig. 4(a), all the samples could be categorized into four different groups. The three kinds of edible fungi are different, and they are also clearly different from the volatile component composition of EUb and their fermentation products. This result was also similar to the previous fingerprint obtained (Fig. 3). For all samples, EUb and their fermentation products were gathered in the same area. The difference between EUb and its fermentation product was not obvious. The samples were further analyzed and the results were shown in Fig. 4 (b). The samples were divided into three areas, which also showed that within a certain range, the volatile composition of EUb raw material and its fermentation product are also different. Compared to other groups of EUb, the GF-EUb-F group was different from raw materials and other fermentation groups, and could also exhibit its own characteristics. In addition, it could be seen from the analysis results that the composition of the volatile components of the three groups of GL-EUB-F, GF-EUB-F, and GF-GF-EUB-F was relatively close. Partial least squares discriminant analysis is a method of supervised comparison, which can reduce the interference of many factors on the analysis and is used to evaluate the differences between different groups. The quality of the PLS-DA model is described by statistical parameters, and the statistical parameters $R^{2} X(0.986$ and 0.905$), R^{2} Y(0.984$ and 0.985$)$ and $Q^{2}(0.825$ and 0.841$)$ were close to 1 in this experiment (Fig. 4). Therefore, the model was reasonable and could accurately describe the differences between different samples.

The volatile metabolites are also considered as phenotypical characteristics of three edible fungi and different samples from EUb. Certainly, the GC-IMS volatile profiles of these samples shared the same volatile compounds but differed in intensity of the signals. This also indicated that different samples have their own unique characteristic volatile components (Gallegos et al. 2017). The volatile components of EUb were changed before and after fermentation. Different fermentation strains had different effects on the composition and content of volatile components of fermentation products.

\section{CONCLUSIONS}

1. The fingerprints of the characteristic volatile components of the three edible fungi, $\mathrm{EUb}$, and the fermentation products were respectively established. A total of 186 signal peaks from topographic plots were observed, and 51 typical target compounds were identified, including alcohols, aldehydes, acids, and esters.

2. Based on the compounds that have been detected, there were differences in the volatile constituents of the three edible fungi. EUb also has its own unique composition of volatile components. After fermentation with different edible fungi, the change of volatile components in the product was different compared to the raw materials.

3. The results of PLS-DA clearly showed that the samples (edible fungi, EUb, and different fermentation product) in a relatively independent space were well distinguished. This further illustrated that the composition and relative content of 
volatile components of EUb could be changed by different microbial strains through bio-fermentation technology.

\section{ACKNOWLEDGMENTS}

This study was financially supported by the GDAS' Project of Science and Technology Development (2019GDASYL-0502003, 2019GDASYL-0103024), the National Natural Science Foundation of China (31660181), and funded by the China Postdoctoral Science Foundation (2019M653099).

\section{REFERENCES CITED}

Akacha, N. B., and Gargouri, M. (2015). "Microbial and enzymatic technologies used for the production of natural aroma compounds: Synthesis, recovery modeling, and bioprocesses," Food Bioprod. Process. 94, 675-706. DOI: 10.1016/j.fbp.2014.09.011

Allen, A., Williams, M. R., and Sigman, M. E. (2019). "Application of likelihood ratios and optimal decision thresholds in fire debris analysis based on a partial least squares discriminant analysis (PLS-DA) model," Forensic Chem 16, 100188

Arroyo-Manzanares, N., Martín-Gómez, A., Jurado-Campos, N., Garrido-Delgado, R., Arce, C., and Arce, L. (2018). "Target vs. spectral fingerprint data analysis of Iberian ham samples for avoiding labelling fraud using headspace-gas chromatography-ion mobility spectrometry," Food Chem. 246, 65-73. DOI: 10.1016/j.foodchem.2017.11.008

Bel-Rhlid, R., Berger, R. G., and Blank, I. (2018). "Bio-mediated generation of food flavors - Towards sustainable flavor production inspired by nature," Trends Food Sci. Tech. 78, 134-143. DOI: 10.1016/j.tifs.2018.06.004

Borsdorf, H., and Eiceman, G. A. (2006). "Ion mobility spectrometry: Principles and applications," Appl. Spectrosc. Rev. 41, 323-375. DOI: 10.1080/05704920600663469

Castro-Muñoz, R. (2019). "Pervaporation: The emerging technique for extracting aroma compounds from food systems," J. Food Eng. 253, 27-39. DOI: 10.1016/j.jfoodeng.2019.02.013

Cavanna, D., Zanardi, S., Dall'Asta, C., and Suman, M. (2019). "Ion mobility spectrometry coupled to gas chromatography: A rapid tool to assess eggs freshness," Food Chem. 271, 691-696. DOI: 10.1016/j.foodchem.2018.07.204

Chouinard, C. D., Wei, M. S., Beekman, C. R., Kemperman, R. H. J., and Yost, R. A. (2016). "Ion mobility in clinical analysis: Current progress and future perspectives," Clin. Chem. 62, 124-144. DOI: 10.1373/clinchem.2015.238840

Fink, T., Baumbach, J. I., and Kreuer, S. (2014). "Ion mobility spectrometry in breath research," J. Breath Res. 8, Article ID 027104. DOI: 10.1088/1752-7155/8/2/027104

Gallegos, J., Arce, C., Jordano, R., Arce, L., and Medina, L. M. (2017). "Target identification of volatile metabolites to allow the differentiation of lactic acid bacteria by gas chromatography-ion mobility spectrometry," Food Chem. 220, 362-370. DOI: 10.1016/j.foodchem.2016.10.022

Gallegos, J., Garrido-Delgado, R., Arce, L., and Medina, L. M. (2015). "Volatile metabolites of goat cheeses determined by ion mobility spectrometry. Potential 
applications in quality control," Food Anal. Method 8, 1699-1709. DOI: 10.1007/s12161-014-0050-1

Garrido-Delgado, R., Del Mar Dobao-Prieto, M., Arce, L., and Valcárcel, M. (2015). "Determination of volatile compounds by GC-IMS to assign the quality of virgin olive oil," Food Chem. 187, 572-579. DOI: 10.1016/j.foodchem.2015.04.082

Gerhardt, N., Birkenmeier, M., Sanders, D., Rohn, S., and Weller, P. (2017). "Resolution-optimized headspace gas chromatography-ion mobility spectrometry (HS-GC-IMS) for non-targeted olive oil profiling," Anal. Bioanal. Chem. 409, 3933 3942. DOI: 10.1007/s00216-017-0338-2

Gerhardt, N., Birkenmeier, M., Schwolow, S., Rohn, S., and Weller, P. (2018). "Volatile compound fingerprinting by headspace-gas-chromatography ion-mobility spectrometry (HS-GC-IMS) as a benchtop alternative to $1 \mathrm{H}$ NMR profiling for assessment of the authenticity of honey," Anal. Chem. 90, 1777-1785. DOI: 10.1021/acs.analchem.7b03748

Hajialigol, S., Ghorashi, S. A., Alinoori, A. H., Torabpour, A., and Azimi, M. (2012). "Thermal solid sample introduction-fast gas chromatography-low flow ion mobility spectrometry as a field screening detection system," J. Chromatogr. A 1268, 123-129. DOI: 10.1016/j.chroma.2012.10.010

He, X., Wang, X., Fang, J., Chang, Y., Ning, N., Guo, H., Huang, L., Huang, X., and Zhao, Z. (2017). "Structures, biological activities, and industrial applications of the polysaccharides from Hericium erinaceus (Lion's Mane) mushroom: A review," Int. J. Biol. Macromol. 97, 228-237. DOI: 10.1016/j.ijbiomac.2017.01.040

He, X., Wang, J., Li, M., Hao, D., Yang, Y., Zhang, C., He, R., and Tao, R. (2014). "Eucommia ulmoides Oliv.: Ethnopharmacology, phytochemistry and pharmacology of an important traditional Chinese medicine," J. Ethnopharmacol. 151, 78-92. DOI: 10.1016/j.jep.2013.11.023

Hirata, T., Ikeda, T., Fujikawa, T., and Nishibe, S. (2014). "The chemistry and bioactivity of Eucommia ulmoides Oliver leaves," Stud. Nat. Prod. Chem. 41, 225-260. DOI: 10.1016/B978-0-444-63294-4.00008-5

Holopainen, S., Luukkonen, V., Nousiainen, M., and Sillanpää, M. (2013). "Determination of chlorophenols in water by headspace solid phase microextraction ion mobility spectrometry (HS-SPME-IMS)," Talanta 114, 176-182. DOI: 10.1016/j.talanta.2013.04.023

Jafari, M. T., Saraji, M., and Sherafatmand, H. (2012). "Design for gas chromatographycorona discharge-ion mobility spectrometry," Anal. Chem. 84, 10077-10084. DOI: $10.1021 / \mathrm{ac} 3025398$

Kanu, A. B., and Hill, H. H. (2004). "Ion mobility spectrometry: Recent developments and novel applications," Spectrom. Techniques 15, 125-136.

Leonhardt, J. W. (2013). "A new ppb-gas analyzer by means of GC-ion mobility spectrometry (GC-IMS),” J. Radioanal. Nucl. Chem. 257, 133-139. DOI: 10.1023/A:1024765814585

Li, L., Guo, Y., Zhao, C., Wei, M., Liu, J., Zu, Y., Wang, F., and Yang, L. (2016). "Microwave-assisted method for simultaneous hydrolysis and extraction for preparation of geniposidic acid from Eucommia ulmoides bark using basic imidazolide ionic liquid," Int. J. Chem. Eng. 2016, Article ID 2135350. DOI: $10.1155 / 2016 / 2135350$ 
Li, M., Yang, R., Zhang, H., Wang, S., Chen, D., and Lin, S. (2019). "Development of a flavor fingerprint by HS-GC-IMS with PCA for volatile compounds of Tricholoma matsutake Singer," Food Chem. 290, 32-39. DOI: 10.1016/j.foodchem.2019.03.124

Liese, A., and Filho, M. V. (1999). "Production of fine chemicals using biocatalysis," Curr. Opin. Biotech. 10(6), 595-603. DOI: 10.1016/S0958-1669(99)00040-3

Limero, T. F., Nazarov, E. G., Menlyadiev, M., and Eiceman, G. A. (2015). "Characterization of ion processes in a GC/DMS air quality monitor by integration of the instrument to a mass spectrometer," Analyst 140, 922-930. DOI: 10.1039/c4an01800a

Márquez-Sillero, I., Cárdenas, S., and Valcárcel, M. (2011). "Direct determination of 2, 4, 6-tricholoroanisole in wines by single-drop ionic liquid microextraction coupled with multicapillary column separation and ion mobility spectrometry detection," $J$. Chromatogr. A 1218(42), 7574-7580. DOI: 10.1016/j.chroma.2011.06.032

Mochalski, P., Wiesenhofer, H., Allers, M., Zimmermann, S., Güntner, A. T., Pineau, N. J., Lederer, W., Agapiou, A., Mayhew, C. A., and Ruzsanyi, V. (2018). "Monitoring of selected skin-and breath-borne volatile organic compounds emitted from the human body using gas chromatography ion mobility spectrometry (GC-IMS), $J$. Chromatogr. B 1076, 29-34. DOI: 10.1016/j.jchromb.2018.01.013

Perl, T., Jünger, M., Vautz, W., Nolte, J., Kuhns, M., Borg-von Zepelin, M., and Quintel, M. (2011). "Detection of characteristic metabolites of Aspergillus fumigatus and Candida species using ion mobility spectrometry-metabolic profiling by volatile organic compounds," Mycoses 54(6), 28-837. DOI: 10.1111/j.14390507.2011.02037.x

Puton, J., and Namieśnik, J. (2016). "Ion mobility spectrometry: Current status and application for chemical warfare agents detection," TrAC Trend. Anal. Chem. 85(Part B), 10-20. DOI: 10.1016/j.trac.2016.06.002

Toraman, H. E., Abrahamsson, V., Vanholme, R., Van Acker, R., Ronsse, F., Pilate, G., Boerjan, W., Van Geem, K. M., and Marin, G. B. (2018). “Application of Py-GC/MS coupled with PARAFAC2 and PLS-DA to study fast pyrolysis of genetically engineered poplars," J. Anal. Appl. Pyrol. 129, 101-111. DOI: 10.1016/j.jaap.2017.11.022

Tzschoppe, M., Haase, H., Höhnisch, M., Jaros, D., and Rohm, H. (2016). "Using ion mobility spectrometry for screening the autoxidation of peanuts," Food Control 64, 17-21. DOI: 10.1016/j.foodcont.2015.12.017

Vajpeyi, S., and Chandran, K. (2015). "Microbial conversion of synthetic and food waste-derived volatile fatty acids to lipids," Bioresource Technol. 188, 49-55. DOI: 10.1016/j.biortech.2015.01.099

Vautz, W., Zimmermann, D., Hartmann, M., Baumbach, J., Nolte, J., and Jung, J. (2006). "Ion mobility spectrometry for food quality and safety," Food Addit. Contam. 23(11), 1064-1073. DOI: 10.1080/02652030600889590

Wang, X., Yang, S., He, J., Chen, L., Zhang, J., Jin, Y., Zhou, J., and Zhang, Y. (2019). "A green triple-locked strategy based on volatile-compound imaging, chemometrics, and markers to discriminate winter honey and sapium honey using headspace gas chromatography-ion mobility spectrometry," Food Res. Int. 119, 960-967. DOI: 10.1016/j.foodres.2019.01.004

$\mathrm{Xu}$, J. W., Zhao, W., and Zhong, J. J. (2010). "Biotechnological production and application of ganoderic acids," Appl. Microbiolol. Biot. 87(2), 457-466. DOI: $10.1007 / \mathrm{s} 00253-010-2576-5$ 
Zhang, L., Shuai, Q., Li, P., Zhang, Q., Ma, F., Zhang, W., and Ding, X. (2016). “Ion mobility spectrometry fingerprints: A rapid detection technology for adulteration of sesame oil," Food Chem. 192, 60-66. DOI: 10.1016/j.foodchem.2015.06.096

Zhao, T., Fan, Y., Mao, G., Feng, W., Zou, Y., Zou, Y., Yang, L., and Wu, X. (2017). "Purification, characterization and antioxidant activities of enzymolysis polysaccharide from Grifola frondosa," Iran. J. Pharm. Res. 16(1), 347-356.

Zhu, M. Q., and Sun, R. C. (2018). "Eucommia ulmoides Oliver: A potential feedstock for bioactive products," J. Agr. Food Chem. 66, 5433-5438. DOI: 10.1021/acs.jafc.8b01312

Zou, N., Yuan, C., Liu, S., Han, Y., Li, Y., Zhang, J., Xu, X., Li, X., and Pan, C. (2016). "Coupling of multiwalled carbon nanotubes/polydimethylsiloxane coated stir bar sorptive extraction with pulse glow discharge-ion mobility spectrometry for analysis of triazine herbicides in water and soil samples," J. Chromatogr. A 1457, 14-21. DOI: 10.1016/j.chroma.2016.06.043

Article submitted: October 18, 2019; Peer review completed: February 22, 2020; Revised version received and accepted: May 7, 2020; Published: July 22, 2020.

DOI: 10.15376/biores.15.3.6941-6959 